\title{
The Formulation of Two-Sex Age Dependent Simulation Model
}

\author{
Jun HAGIHARA ${ }^{1}$ and Ryutaro OHTSUKA ${ }^{1}$
}

This study developed the stochastic simulation model constructed on the unit which was categorized by subgroups of age, sex and marital status, and evaluated the validity of the model. Events in the model were birth, death, and marriage. The estimation of the parameters in a death and marriage model was done by using vital statistics data from Japan. The desired family size and the proportion of contraceptive user at the time when a mother reached the desired family size were incorporated in the birth model. Occurrence of an event was determined for each individual in subgroups using a uniform random number based on the age-specific probability for the specified events. A series of sensitivity analysis was performed to evaluate the model. Results showed that the model was robust across the initial population sizes. When the effects of changes in the desired family size and the contraceptive use on population growth were considered, both affected the population growth rate. Similarly, changes of the age-specific mortality rate and the age-specific marriage rate also affected the population growth. From the results of these sensitivity analyses, it was concluded that this simulation model was an effective tool for analysis of reproductive demography.

Key words : proximate determinants, fertility, simulation, two-sex model

\section{Introduction}

Age dependent dynamics of human populations have been investigated in many fields, such as demography, epidemiology, and biomathematics. Historically, one-sex model for elucidating population increase and age distribution was first formulated by Sharpe and Lotka (1911), and since then a number of onesex models have been developed. However, there are two drawbacks in these models when they treat empirical data of the identical population, independently for males and females (Pollard, 1997). First, the number of population exponentially increases. Second, the "net reproduction rates" of males and females, i.e. the average number of sons who survive to reproductive age per male for the former and the average number of daughters who survive to reproductive age per female for the latter, are not identical even though the source population is identical. This discrepancy attributes mostly to disregard of marriage. It is thus necessary to distinguish between both sexes and to incorporate their age distribution and age dependent process of marriage into the simulation model. 
The stochastic simulation analysis for reproduction has mainly focused on models of fertility. A basic microsimulaiton model about fertility was developed by Barret (1971). Since then, various microsimulation studies have depended on this framework to extensively evaluate methods for measuring various demographic processes. For example, the methods proposed by Coale and Trussell (1974) and David et al. (1988) were effective for identifying the effects of fertility control on population dynamics, even though data about the use of birth control were lacked (Okun, 1994). However, these microsimulation analyses, including Barret's method, do not incorporate other processes, such as marriage and death, that affect the population size and contraceptive behavior that reflects female's (or couple's) desired number of children.

Mode and Salsbulg (1993) developed stochastic simulation model which incorporated marriage process. The simulation model developed in the present study revised their model, considering several essential points. First, contraceptive prevalence and desired family size were incorporated as the variables. Second, since their model had difficulties for implementing death components and estimating parameters from empirical data, variables of death components were incorporated in this model, following Denny's (1997) model. In other words, the major efforts for this study were devoted to making a new model that bridged the two models, i. e. Mode and Salsbulg's model and Denny's model, and incorporated contraceptive behavior, paying attention to applicabilities to various populations.

\section{Methodology}

\section{Framework of the Simulation Model}

This simulation model computed the projection using stochastic process in discrete time. Each individual in this simulation model is exposed to vital events every year. and whether a vital event (birth, death, or marriage) occurs or not is determined, using age-specific probability.

Age is denoted by $x$, which takes nonnegative integers equal to or less than, $r$, which is assumed as the highest age. In this simulation, all individuals of each age are categorized into three: unmarried males, unmarried females, and married couples that combine males and females. Newborns entering the population are represented as age 0 . This categorization system by married or unmarried age/sex groups followed Mode and Salsburg (1993). Let $m_{i}$ represent the number of unmarried males with age $i=0,1,2 \ldots r$, and $f_{j}$ the number of unmarried females with age $j=0,1,2 \ldots r$. For the married group, let $c_{i j}$ represent the number of married males with age $i$ and females with age $j$. Then, $m_{i}$ and $f_{j}$ are decreased by death or marriage and increased by couple dissolution due to death of a partner in one year of the simulation. In the same manner, $c_{i j}$ is decreased by couple dissolution due to death of a partner and increased by couple formation.

Figure 1 shows the flow diagram of the simulation program, consisting of six sections. For convenience of debugging and alteration, a sub-routine program is incorporated in each of the death, marriage and birth sections, which are described later in detail. Table 1 shows the principal variables in the simulation program, which was written in $\mathrm{C}$ 


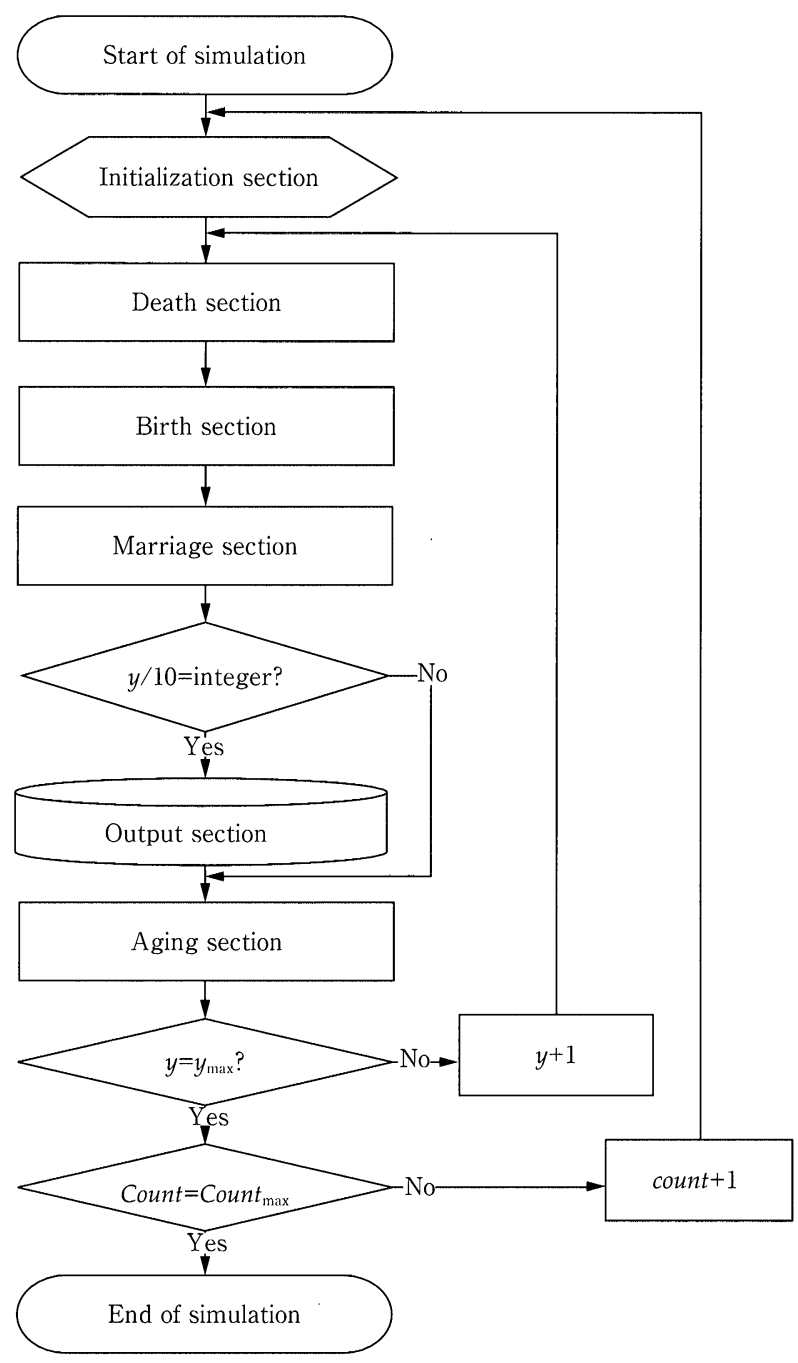

Fig. 1 The flow diagram of simulation.

Table 1 Principal variables used in this simulation

\begin{tabular}{ll}
\hline \multicolumn{1}{c}{ Variables } & \multicolumn{1}{c}{ Meaning } \\
\hline$y$ & The year in the simulation \\
$y_{\max }$ & The duration of run $(=100$ in this study $)$ \\
Count & The number of repetitions for each parameter set \\
Count $\max$ & The maximum number of Count $(=100$ in this study $)$ \\
$m_{i}$ & The number of unmarried males at age $i(0 \leq i \leq 100)$ \\
$f_{j}$ & The number of unmarried females at age $j(0 \leq j \leq 100)$ \\
$C_{i j}$ & The number of married couples consisting of males with age $i$ and \\
\hline
\end{tabular}


language and run on Linux OS. Converting probability to the occurrence of events was determined according to uniform random number sequence and standard normal random number sequence, which were generated by the algorithm developed by Press et al (1993) and Park and Miller (1988).

\section{The Parametric Components of the Simu- lation Model}

Three parametric models, each representing mortality for either sex, fertility, and marriage, generate age-specific probabilities by means of occurrence or non-occurrence of each event in the simulation model.

The mortality component of the simulation model is based on a three-parameter model described by Denny (1997), as follows;

$$
l(x)=\frac{1}{1+a(x /(105-x))^{3}+b\left(e^{x /(105 \cdot n)}-1\right)^{1 / 2}+c\left(1-e^{-2 x}\right)}
$$

where, $l(x)$ means survivorship function. It has been proved to fit well to the survivorship function in many life tables of either sex in a wide range of mortality levels, and the three parameters, $a, b$, and $c$, in the equation (1) are indicators of mortality in the late, middle, and early periods of life span, respectively (Denny, 1997).

It is known that parameters, $a, b$, and $c$, in this formula, should not be expected to be negative values by the mathematical definition, which tends to decline with increase in life expectancy (Denny, 1997). The death component in the simulation model needs the probability of survival, denoted by $p(x)$ in life table analysis, in discrete time of year. To implement this parametric model (equation (1)) to the death component, it is transformed by the following formula;

$$
p(x)=l(x)-l(x+1) .
$$

The fertility component of this model contains three subcomponents, i.e. fecundability, sterility, and marriage. In this simulation model, it is assumed that birth occurs only in the women who are married.

Each woman's initial fecundability by age is determined as follows:

$$
f(x)=\frac{x^{(-1}(1-x)^{\beta-1}}{B(\alpha, \beta)}
$$

where, $B(a, \beta)$ is the beta distribution function shown below;

$$
B(\alpha, \beta)=\int_{0}^{1} x^{u-1}(1-x)^{\beta-1} d x .
$$

This formula is a probability density function, and thus does not represent absolute line of the age-specific fecundity. Thus, the formula must be multiplied by 16 , which is assumed as the maximum potential value of a woman's births (Bongaarts et al., 1987).

At the start of each year, it is determined whether a woman becomes sterile by using sterility function developed by Pittenger (1973), as follows;

$$
\begin{aligned}
& s(x)=1.01155-\exp \left[-k\left(r^{x-12}-1\right) \ln (r)\right], \\
& 12 \leq x \leq 50 .
\end{aligned}
$$

This model determines the end of women's reproductive function, following Trussell and Olsen (1983). In other words, this model af- 
fects only the end of reproductive life. The decline of fertility by artificial sterility (e.g. contraception) is described in the birth section.

There are various parametric distributions which fit to data of the age at the first marriage in cohorts of females and males. Among them, a lognormal distribution by Mode (1985) was applied to this study. Let $\phi$ be the distribution function of the standard normal distribution, let $X$ be a random variable representing age at the first marriage, and let $a_{0}$ be the youngest age at marriage. Let $p$ be the proportion of individuals who eventually marry, and let $\mu$ and $\sigma^{2}$ be the mean and variance of normal distribution, and then $G(x)$ can be computed by the formula;

$$
G(x)=p \phi\left(\frac{\ln \left(x-a_{0}\right)-\mu}{\sigma}\right) .
$$

Because this simulation model needs discrete time risk function associated with equation (5), this formula was transformed to;

$$
\mathrm{g}_{0}\left\{\begin{array}{l}
=0 . \\
=p \frac{1}{\sqrt{2 \pi}\left(x-a_{0}\right) \sigma} \exp \left[-\frac{1}{2}\left(\frac{\ln _{\mathrm{e}}\left(x-a_{0}\right)-\mu}{\sigma}\right)^{2}\right] \\
\left(x \geq a_{0}\right)
\end{array}\right.
$$

\section{Description of Each Section}

In the initialization section, the number of each age/sex group calculated by $l(x)$ in Equation (1) and an initial population size, is given before simulation runs.

Next, the death section consists of two subsections, one for the unmarried group and the other for the married group. In the unmarried group's subsection, if the uniform random number generated in $(0,1]$ for each unmarried male or female is smaller than age-specific death rate in Equation (2), then he or she dies, and $m_{i}$ or $f_{i}$ becomes $m_{i}$ or $f_{j}$ -1 . This procedure is repeated $m_{i}-1$ or $f_{i}$ times from $i(j)=0$ to $i(j)=100$.

In the married group's subsection, the first process is to count the decreased number of couples of male with age $i$ and female with age $j$ (denoted as $c_{i j}$ ) due to death of either male or female; among the $c_{i j}$, the number of died males with age $i$ (defined as $d m_{i}$ ) and that of died females with age $j\left(d f_{j}\right)$ are separately calculated in the same procedure as for the unmarried group. When $d m_{i}-d_{f j}$ (denoted as $d t$ ) is positive, $c_{i j}$ is reduced to $c_{i j}-$ $d m_{i}$ and $f_{i}$ increases to $f_{i}+d m_{i}-d f_{j}$. Otherwise (when $d t$ is negative), the same process, in which $m_{i}$ is replaced by $f_{j}$ and vice versa, is carried out. This procedure is repeated $i \times$ $j$ times.

Because of the assumption that only married females give birth in this simulation model, birth section treats only married group, denoted as $c_{i j}$. The flow diagram of birth section is shown in Figure 2. This section determines whether the reproductive status of a female is sterile by comparing age-specific sterility rate generated by Equation (4) with the uniform random number generated in $(0,1]$. If she is sterile, her birth process is terminated. Otherwise, it is determined whether she practices the contraception or not. If her parity exceeds the given desired family size, a random number is generated in $(0,1]$ and if the random number is smaller than the given rate of use of contraception, birth process is treated as controlled 


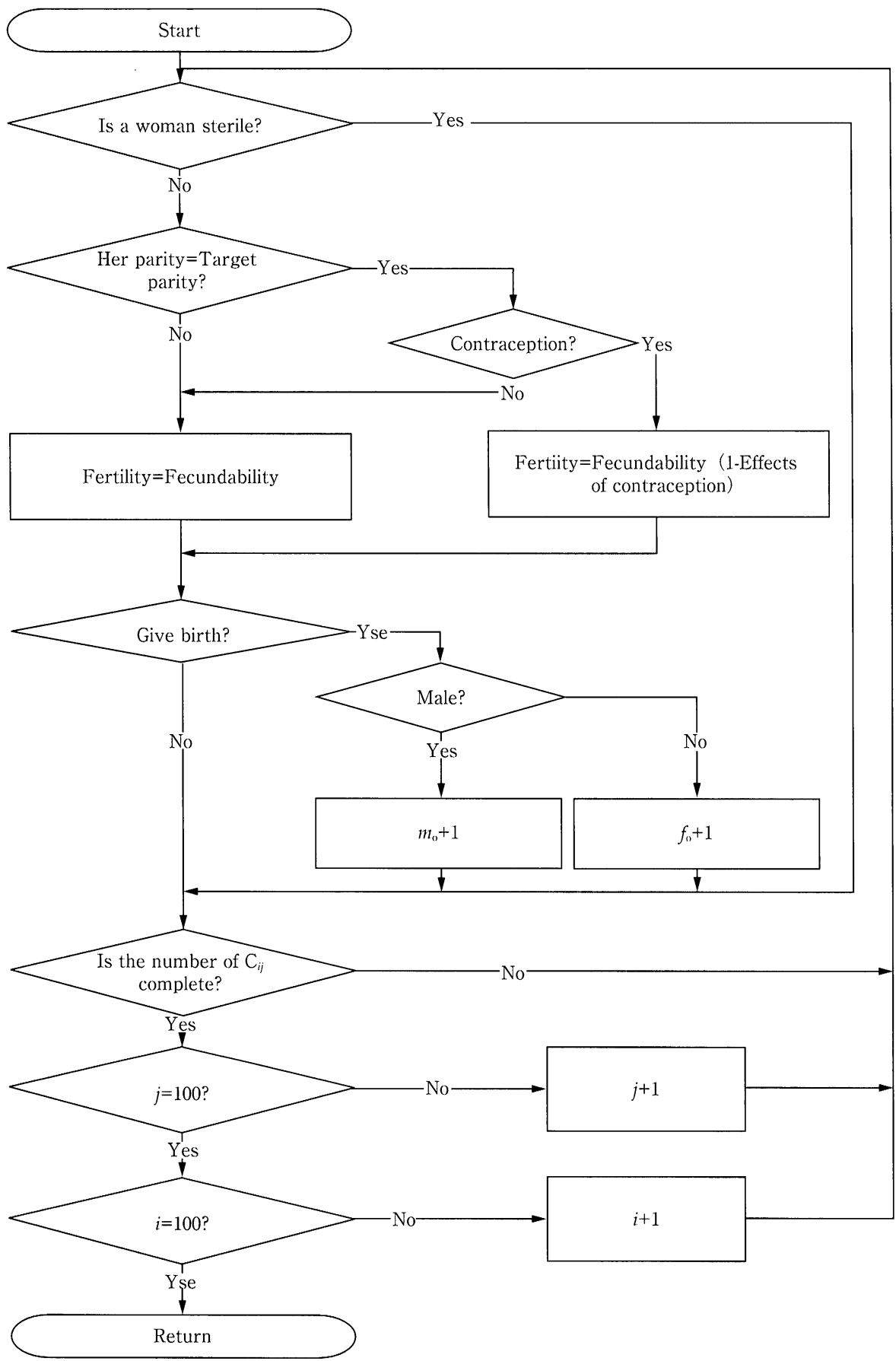

Fig. 2 The flow diagram of birth section. 
process; otherwise, the probability of conception in the year $\mathrm{pm}$ is same as age-specific fecundability (Equation (3)). In the controlled process, given that the effect of contraception is denoted as $e i$, contraception lowers the probability of conception in the year from $p m$ to $p m(1-e i)$. Value of $e i$ used in this study was 0.93 , which reflected the prevailed effect of condom use (Okun, 1994). Therefore, the probability of conception lowers by $7 \%$ of age-specific fecundability by using the condom. If the uniform random number generated in $(0,1]$ is smaller than age-specific probability of conception, she bears one child in that year. The sex of child is determined as follows; if the uniform random number generated in $(0,1]$ is smaller than the given sex ratio, then the child's sex is determined as male and $m_{0}$ becomes $m_{0}+1$, and otherwise, $f_{0}$ becomes $f_{0}+1$. The above procedure is repeated $c_{i j}$ times and the whole procedure is again repeated $i \times j$ times. This procedure to determine age-specific probability of conception was referred to previous studies of demographic simulation analysis (e.g. Okun, 1994; Trussell and Olsen, 1983).

In the marriage section, if the uniform random number generated in $(0,1]$ is smaller than $g_{0}(x)$ specified in Equation (6), the male marries with one from unmarried females of age, ranging from five years younger or older than him. Standard normal random number, which truncated to an integer denoted as $z$, for age difference within the couple, is generated in $(-5,5)$. If $f_{i+z}$ is equal to 0 , marriage is not formed. If the male cannot find a wife in five trials, then he fails to marry in this year. When $f_{i+z}$ is not equal to 0 (the male can find a wife), then $m_{i}$ becomes $m_{i}-1$ and $f_{i+z}$ becomes $f_{i+z}-1$, and $c_{i i+z}$ be- comes $C_{i i+z}+1$. The above process is repeated $m_{i}$ times, from $i=a_{0}$ in Equation (6) to $i=$ 100.

In the output section, the results of the three sections (death, birth, and marriage) are transferred to the hard-disk drive of computer. This section is worked once a year.

In the final section for aging, one year is added to age of each person, who belongs to either unmarried or married group; values of $m_{i}, f_{j}$, and $c_{i j}$ change to $m_{i+1}, f_{j+1}$, and $c_{i+1 j+1}$, respectively. The number of newly born male and female children in the preceding year are treated as $m_{0}$ and $f_{0}$.

After aging section, 1 is added to $y$, the simulation year. If $y$ is not equal to $y_{\max }$ (this value is given before the simulation run), the simulation program returns to death section. When $y$ is equal to $y_{\max }$, one run is over and the next run commences. This process is repeated until count is equal to count $t_{\max }$, which is given before the simulation run.

\section{Variables and Parameters}

The following parameters in the simulation model are given from empirical settings: $a, b$, and $c$ in Equation (1), $a$ and $\beta$ in Equation (3), $k$ and $r$ in Equation (4), and $a_{0}, \mu$ and $\sigma^{2}$ in Equation (6). Other parameters in the simulation model are variables.

The principal variables, in the abbreviated form, used in the simulation model in this study are shown in Table 1 . The variables $y$ and count refers to the year in the simulation and the number of repetition of one parameter set, respectively. The duration of run $\left(y_{\max }\right)$ was 100 years and the number of runs for each parameter set (count $t_{\max }$ ) was 100. 
Table 2 Parameters in birth component

\begin{tabular}{lll}
\hline \multicolumn{1}{c}{ Parameters } & \multicolumn{1}{c}{ Meaning } & Value \\
\hline$a, b$ in equations $(2)$ and $(3) * 1$ & Parameters determining the shape of fecundability. & 2,8 \\
$k, r$ in equation $(4) * 2$ & Parameters determining the age-specific sterility rate. & $0.0002,1.251242$ \\
Desired family size & The number of children that a woman desires to have. & $3,4,5$ \\
Effectiveness of contraception $* 3$ & The rate of reducing the fecundability. & 0.93 \\
Contraceptive rate $* 1$ & The rate of using contraception when a woman achieves her & $0.5,0.7,0.9$ \\
& desired family size. & \\
\hline
\end{tabular}

*1 Source; Okun (1994)

*2 Source; Trussell and Olsen (1983)

*3 This value is assumed to the rate using condoms (Okun, 1994).

Table 3 Parameters of the regression equation from life tables in Japan

\begin{tabular}{clccccc}
\hline \multirow{2}{*}{ Year } & \multirow{2}{*}{ Sex } & \multirow{2}{*}{$e_{0}^{* 1}$} & \multicolumn{3}{c}{ Expected values*2 } & \multirow{2}{*}{ Mean Square error } \\
\cline { 4 - 6 } 1930 & & & $a$ & $b$ & $c$ & 0.00012 \\
& Male & 44.8 & 0.31967 & 0.40705 & 0.26475 & 0.00009 \\
\cline { 4 - 6 } 1990 & Female & 46.5 & 0.10481 & 0.56930 & 0.16027 & 0.00006 \\
& Male & 75.9 & 0.05274 & 0.02669 & $0 * 3$ & 0.00002 \\
\hline
\end{tabular}

*1 $\mathcal{c} 0$ means the expectancy of life at birth.

*2 Expected values were parameters in Equation(1). These were estimated by using non-liner regression.

*3 The value of 0 was fixed because the negative value was generated when parameters were estimated by using non-liner regression.

To estimate parameters in the death component, vital statistics data from Japan in 1930 and 1990 (Statistics Bureau and Statistics Center, 2000) were used. Parameter estimate itself required non-linear regression which was done by the JMP Software Version 4 (SAS Institute, 2000). The reason for selecting these data was that the parameters with wide ranges were necessary to evaluate the simulations in the settings of both developed and developing countries. The procedure of estimating parameters consisted of two steps. First, an abridged life table was calculated from age-specific death rates in 1930 and 1990. Second, the parameters, $a, b$, and $c$, in Equation (1) were estimated using non-linear regression on $l(x)$ in the abridged life tables.

The parameters in the marriage component were estimated using non-linear regression on age-specific rates of first marriage by vital statistics in Japan. To estimate the parameters in the marriage component, non-linear regression, which was same as that for estimating the parameters in the death component, was used.

Parameters and variables used in the birth section are shown in Table 2. The parameters of beta distribution ( $\alpha$ and $\beta$ in Equation (3')), which assumed age-specific fecundability rate, were determined as 2 and 8, corresponding to the usual levels among noncontracepting young cohabiting women (Okun, 1994). 


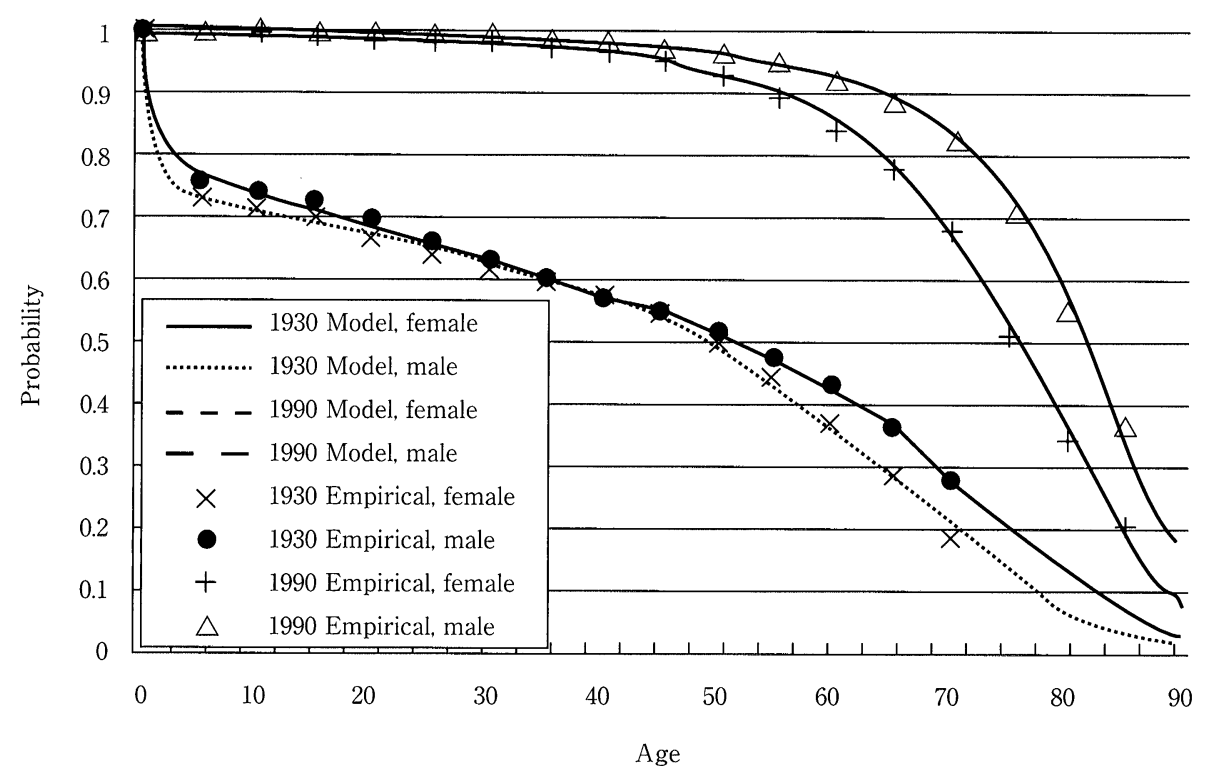

Fig. 3 The observed and expected values of $I(x)$ for Japanese vital registration.

Table 4 Estimated parameters in the marriage function in Japan

\begin{tabular}{ccccc}
\hline \multirow{3}{*}{ Year } & \multicolumn{3}{c}{ Expected values*1 } & \multirow{2}{*}{ Mean square error } \\
\cline { 2 - 4 } & $a_{0}$ & $\mu$ & $\sigma$ & 0.00019 \\
\hline 1930 & 18 & 1.643 & 0.675 & 0.00077 \\
1990 & 18 & 1.872 & 0.599 & \\
\hline
\end{tabular}

*1 Expected values were parameters in Equation (6); these were extimated by using non-linear regression.

The parameters $k$ and $r$ in Equation (4) were determined as 0.0002 and 1.251242 , respectively; these values were suggested to be reasonable for most Westernized or developed societies (Pittenger, 1973).

Other variables in the birth component used are shown in Table 2 ; these values were typical for empirical population settings (Okun, 1994). Among the variables in the birth component in Table 2, desired family size ranged from 3 to 5 and contraception rate from 0.5 to 0.9 .

\section{Results}

\section{Results of Parameter Estimates of Each Parametric Model}

In the death component, parameters and other statistical values about goodness of fit are shown in Table 3. Figure 3 graphically depicts the results which are presented in Table 3. For the 1990 Japanese, the best matched estimate of $c$ in Equation (1) had a negative value. Since the parameters of Equation (1) do not have negative value by definition, $c=0$ was used, and other parameters were re-estimated in such cases. 


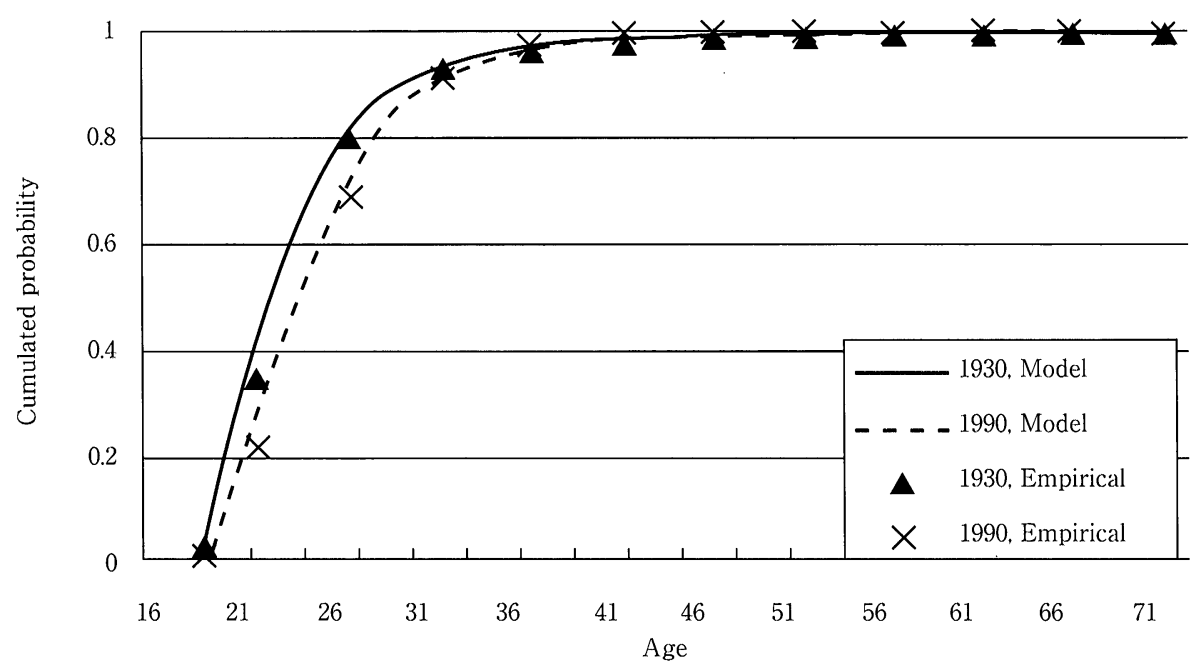

Fig. 4 The observed and expected values of marriage function in Japanese.

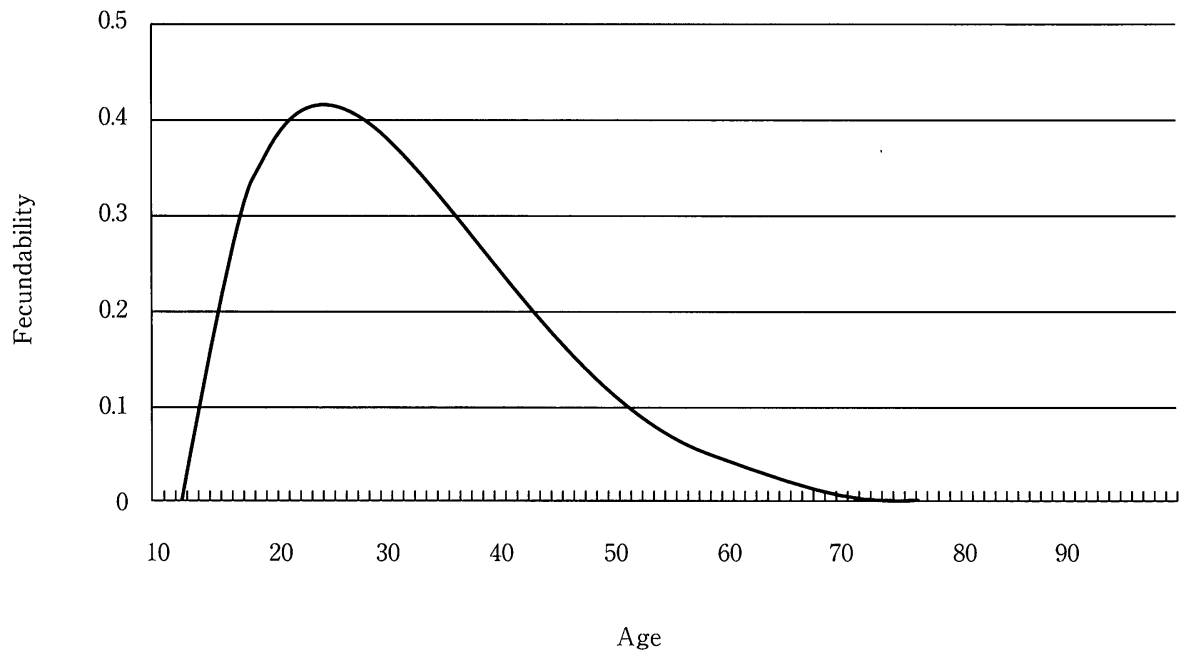

Fig. 5 Age-specific fecundability rate used in the simulation.

For the marriage function described in Equation (6), estimated parameters and statistical value are shown in Table 4 and graphical plot using these parameters are illustrated in Figure 4. These parameters were estimated by Japanese vital statistics data in 1930 and 1990.

In the birth component, age-specific fe- cundability is shown in Figure 5, and agespecific sterility rate, which means the rate for couples to become sterile in that age, is shown in Figure 6.

Effects of Initial Population Size and Birth Settings

At first, the effect of initial population size 


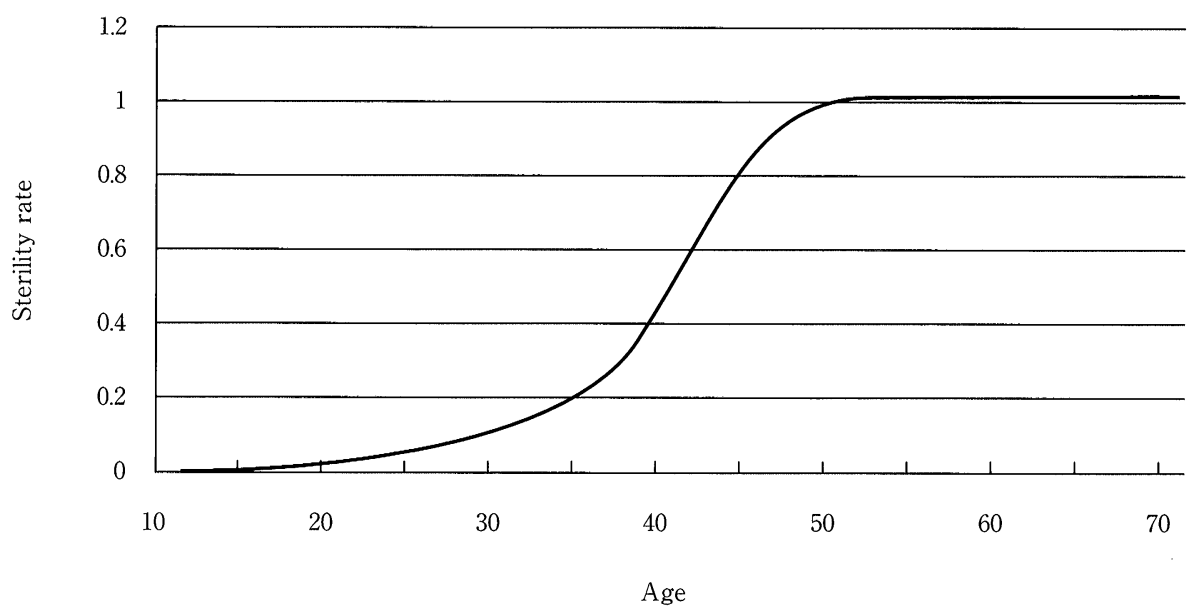

Fig. 6 The age-specific sterility rate.

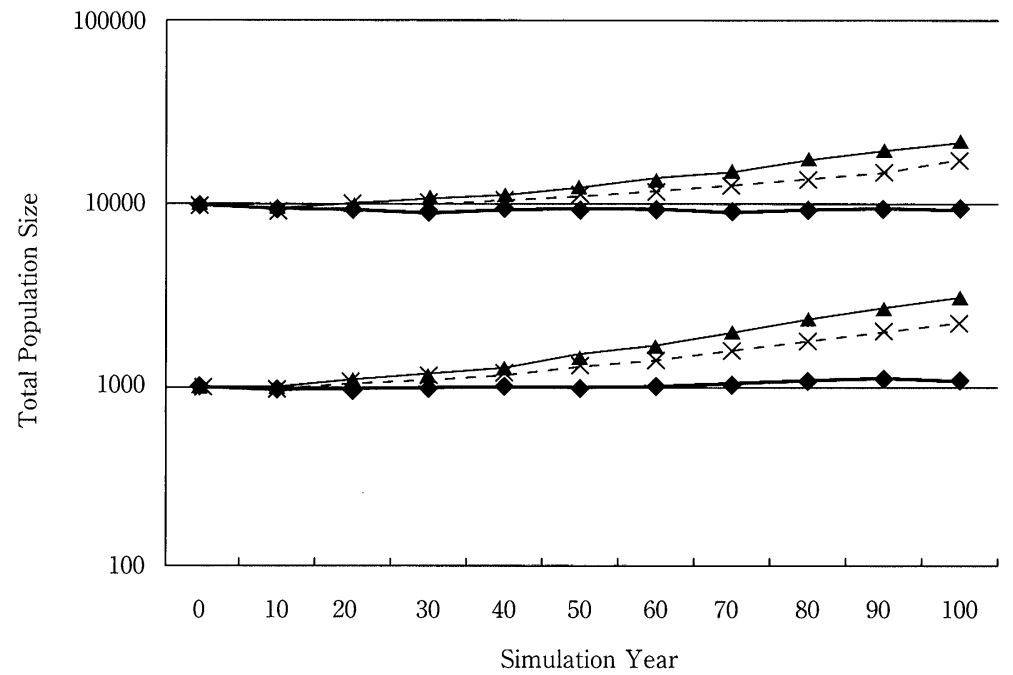

Fig. 7 Effects of initial population size on the simulation.

The common features of this simulation are based on vital statistics data in 1990. Other parameters of birth component are as follows; $-\mathbf{\Delta - D e s i r e d ~ f a m i l y ~ s i z e : ~} 5$, contraception rate: 0.5 . $--X--$ Desired family size: 4 , contraception rate: 0.7 .

$\longrightarrow-$ Desired family size: 3 , contraception rate: 0.9 .

on the result of simulation was investigated. The parameters in the death and marriage components which were derived from Japanese data in 1930 were applied to evaluate the effects because these data were available for wide-ranged age-specific mortality rate due to high mortality.

The results are illustrated in Figure 7. When all parameters except the initial population size were the same, the change of total 


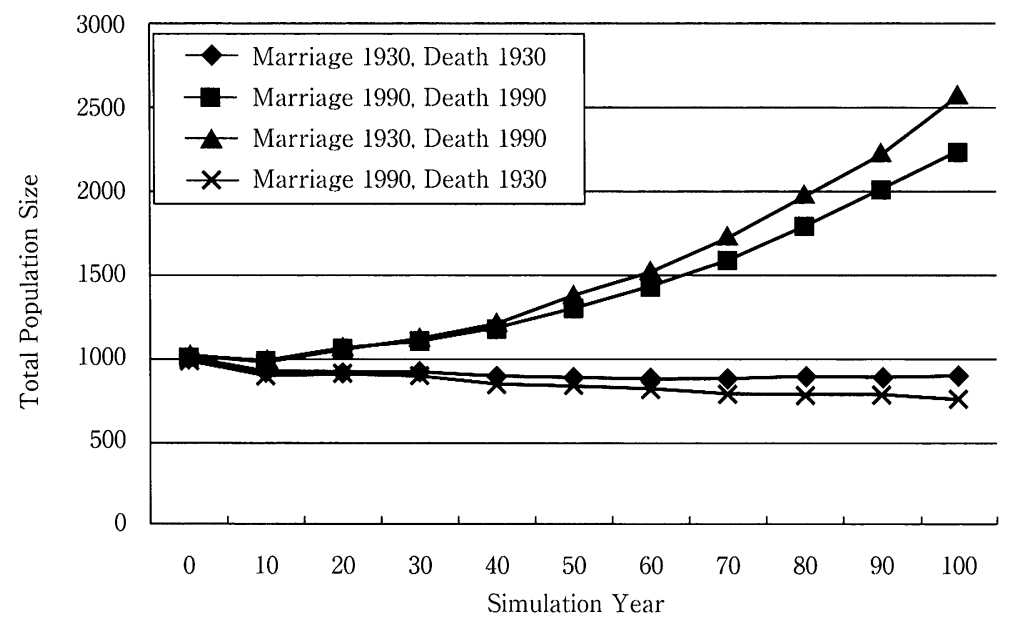

Fig. 8 Effects of death and marriage on the simulation.

Numbers in the legend are the years of data sources.

population size with time was almost identical. This phenomenon was commonly found in all settings, implying that the difference in the initial population size did not considerably affect growth rate of population. It is demonstrated that the simulation model developed in this study is robust when the initial population size is up to 1000 .

Effects of the birth settings on total population size are also shown in Figure 7. When the initial population size was the same, the degree of population growth depended on two variables in the birth setting, i.e. desired family size and contraception rate, both of which functioned after women reached their desired family size. Regarding the effects of the desired family size, population growth was more rapid when this variable was large than when it was small. Regarding contraception rate, population growth was more rapid when this variable was small than when it was large.

\section{Effects of Initial Settings of Death and Marriage Functions}

To investigate effects of settings of death and marriage on total population size, simulation experiments were conducted by exchanging the settings in the marriage component in 1990 for those in 1930, while the settings in the death component were not changed. Other parameters and variables of the simulation were same for all settings: the initial population size was 1000 , duration of simulation was 100 year, the number of repeats of one parameter set was 100 , the desired family size was 4 , and the proportion of contraceptive users at the time when a mother reached the desired family size was $70 \%$.

Figure 8 shows the results of simulation runs. Differences in both death and marriage components affected the population growth (Figure 8). However, the extent of the effects was greater in the death component than in the marriage component. This causation comes from the fact that the difference in 
mortality between 1990 and 1930 was more prominent than that in marriage rates between the two years (see Figures 3, 4).

\section{Discussion}

The present study constructed a new simulation model, which was based on Mode and Salsbulg (1993), but included revisions and additions. Discussion begins with evaluation of performance of this model, using sensitivity analysis.

This study simulated two population settings that were different in both marriage and death structures. The results demonstrated that the death model developed by Denny (1997) fitted well in all data used here (Table 3). It is easier to implement the death model to the simulation and to estimate the parameters when the number of parameters is small than when it is large. While Denny's model has only three parameters, Mode-Jacobson model (Mode and Jacobson, 1984), and Heligman-Pollard model (Heligman and Pollard, 1980) uses, respectively, 10 and eight parameters. Since an undefined value was estimated from Japanese vital statistics in 1990, values of parameter were re-estimated so that the fixed value of 0 was given when the parameters were estimated in Denny's model. Even if these procedures were carried on, the goodness of fit fell little. In the points of the ease of implementation and the high fit to empirical data, it might be concluded that Denny's model is appropriate to be implemented in simulation.

It is also emphasized that age-specific rate of marriage fitted well to the empirical settings in Japan. In addition to the simulation model developed by Mode and Salsburg (1993), the model of the present study incor- porated variables in relation to fertility, which were sterility rate, desired family size, and rate of contraception in the birth component. This procedure that represented agespecific marital fertility rate as a probability of subtracting many factors about proximate determinants from fecundability was the same as previous demographic microsimulation analyses (e.g. Barret, 1971; Okun, 1994; Reinis, 1992; Guinnane et al., 1994).

From the results of sensitivity analysis, it is demonstrated that total population size computed by this simulation model is scarcely affected by the difference in the initial population size, and that total population size computed by this simulation model is prominently affected by differences in the death, marriage, and birth components. It is thus concluded that this simulation model is effective for analysis of reproductive demography.

In this experiment, however, mortality had greater effects on total population size than marriage did. This thought to come from marital age slightly differed between 1930 and 1990 whereas mortality changed considerably from 1930 to 1990 . It is also noted that this simulation model may not completely represent the actual population reproduction process in several respects. For example, there is evidence that married individuals, particularly males, have higher survival rates than unmarried individuals, so-called "marriage protection" (Hu and Goldman, 1990). In addition, this model neglects couple dissolutions due to separation and divorce, which are found in real populations.

\section{Conclusion}

This study developed a new microsimula- 
tion model that was an effective tool to analyze determining factors of fertility and proximate determinants, by means of use of empirical data. This model was characterized by involvement of both vital events (i. e. birth, death, and marriage) and variables in relation to decision-makings of individuals that depended on parity and contraceptive prevalence. In other words, this model differed from some previous models on fertility, which did not include such processes as marriage, and some others which did not include such variables as contraceptive behavior and contraceptive prevalence.

\section{Acknowledgment}

Thanks are due to Dr. Minato Nakazawa, who gave many valuable comments for the manuscript.

\section{References}

Barret JC (1971): Use of a fertility model to refine simulation techniques. Demography, 8(4), 481490

Bongaarts J (1978): A framework for analyzing the proximate determinants of fertility. Population and Development Review, 4(1), 105-132

Bongaarts J, Burch TK, Wachter KW (1987): Family Demography. Oxford University Press (New York)

Coale AJ, Trussell TJ (1974): Model fertility schedules: Variations in the age structure of childbearing in human populations. Population Index, 40, 185-258

David PA, Mroz TA, Sanderson KW, Wachter WC, et al. (1988): Cohort parity analysis: Statistical estimates of the extent of fertility control. Demography, 25(2), 163-188

Denny C (1997): A model of the probability of survival from birth. Mathematical and Computer Modeling, 26(6), 69-78

Guinnane TW, Okun BS, Trussell J (1994): What do we know about the timing of fertility transitions in Europe? Demography, 31(1), 1-20
Heligman L, Pollard JH (1980): The age pattern of mortality. Journal of institute Actuaries, 107, 49-80

Hu Y, Goldman N (1990): Mortality differentials by marital status: An international comparison. Demography, 27, 233-250

Mode CJ (1985): Stochastic Processes in Demography and Their Computer Implementation. Springer-Verlag (New York)

Mode CJ, Jacobson ME (1984): A parametric algorithm for computing model period and cohort and human survival functions. International Journal of Biomedical Computing, 15. 343-56

Mode CJ, Salsburg MA (1993): On the formulation and computer implementation of an agedependent two-sex demographic model. Mathematical Biosciences, 118, 212-40

Okun BS (1994): Evaluating methods for detecting fertility control: Coale and Trussell's model and cohort parity analysis. Population Studies, 48, 193-222

Park SK, Miller KW (1988): Random number generators: Good ones are hard to find. Communications of the ACM, 31, 1192-1201

Pittenger D (1973): An exponential model of female sterility. Demography, 10(1), 113-121

Pollard JH (1997): Modelling the interaction between the Sexes. Mathematical and Computer Modeling, 26(6), 11-24

Press WH, Flannery BP, Teukolsky SA, Vetterling WT (1993): Numerical recipes in C. Gijutu Hyouron sha (Tokyo)

Reinis KI (1992): The impact of the proximate determinants of fertility: Evaluating Bongaarts's and Hobcraft and Little's methods of estimation. Population Studies, 46(2), 309-326

Sharpe FR, Lotka AJ (1911): A problem in age distribution. Philosophical Magazine 21, 435438

Statistics Bureau and Statistics Center (2000) Japan Statistical Yearbook. Nihon Tokei Kyokai (Tokyo)

Trussell J, Olsen R (1983): Evaluation of the Olsen technique for estimating the fertility response to child mortality. Demography, 20(3), 391-405

(Received 3. 30, 2001 ; Accepted 5. 18, 2001) 